Pacific Journal of Mathematics

FACIAL DECOMPOSITION OF LINEARLY COMPACT
SIMPLEXES AND SEPARATION OF FUNCTIONS ON CONES 


\title{
FACIAL DECOMPOSITION OF LINEARLY COMPACT SIMPLEXES AND SEPARATION OF FUNCTIONS ON CONES
}

\author{
L. AsImow AND A. J. Ellis
}

\begin{abstract}
Necessary and sufficient conditions for a linearly compact simplex $K$ to be uniquely decomposable at a face are given. If $P$ is a cone having the Riesz decomposition property and if $-f, g$ are subadditive homogeneous functions on $P$ with $f \geqq g$ then it is shown that there is an additive homogeneous function $h$ on $P$ with $f \geqq h \geqq g$. If $P$ is a lattice cone for the dual space of an ordered Banach space $X$ and if $-f, g$ are also $w^{*}$-continuous then, under certain conditions, it is possible to choose $h \in X$; a consequence of this result is Andô's theorem, that an ordered Banach space has the Riesz decomposition property if its dual space is a lattice. A nonmeasure theoretic proof of Edwards' separation theorem for compact simplexes is also deduced from these results.
\end{abstract}

Let $K$ be a linearly compact simplex in a real vector space $E$. Without loss of generality we will assume that $K$ is contained in a hyperplane $e^{-1}(1)$ and that $E=\operatorname{lin} K$, where lin $K$ denotes the linear hull of $K$. Then it is well known that $E$ is a vector lattice relative to the cone with base $K$, and that co $(K \cup-K)$ is the closed unit ball for a norm making $E$ a pre- $A L$-space. In fact if $K$ is compact for a locally convex Hausdorff topology on $E$ then $E$ is the Banach dual space of $A(K)$, the space of all affine continuous functions on $K$ with supremum norm (cf. [5]). (We refer to [10] as a general reference for the lattice theory and terminology that is used.)

The set $K$ is said to be decomposable at a face $F$ if there exists a complementary face $F^{\prime}$ of $K$ such that $F \cap F^{\prime}=\phi$ while co $\left(F \cup F^{\prime}\right)$ $=K$. If a complementary face to $F$ exists then it is evident that it is uniquely determined; moreover, in this case, Alfsen [1] has shown that the decomposition is unique in the sense that each $k \in K$ has a unique decomposition $k=\lambda x+(1-\lambda) y$ with $x \in F, y \in F^{\prime}$ and $0 \leqq \lambda \leqq 1$. Alfsen has also given a necessary and sufficient condition for $K$ to be decomposable at $F$; we give here other necessary and sufficient conditions which are perhaps more closely tied to the order and norm structure of $E$.

THEOREM 1. Let $K$ be a linearly compact simplex and $F, F^{\prime}$ disjoint faces of $K$. Then $F$ and $F^{\prime}$ are complementary faces for $a$ 
(necessarily unique) decomposition of $K$ if and only if $E$ is the order-direct sum of $\operatorname{lin} F$ and lin $F^{\prime}$. Consequently, if $E$ is complete in its norm then $K$ is uniquely decomposable at $F$ if and only if $F$ is norm-closed.

Proof. Since $F$ is a face of $K$ it is easily verified that lin $F$ is a lattice ideal in $E$ and that (lin $F) \cap K=F$. If $E$ is the orderdirect sum of $\operatorname{lin} F$ and lin $F^{\prime}$ then each $x \in K$ has a unique decomposition $x=y+z$ with $y, z \geqq 0, y \in \operatorname{lin} F, z \in \operatorname{lin} F^{\prime}$; hence $K=$ co $\left(F \cup F^{\prime}\right)$, and the decomposition is unique.

Suppose conversely that $K=\operatorname{co}\left(F \cup F^{\prime}\right)$. Then, since $E=\operatorname{lin} F$ $+\operatorname{lin} F^{\prime}$, it will follow that $E$ is the order-direct sum of $\operatorname{lin} F$ and lin $F^{\prime}$ if we prove that $\operatorname{lin} F^{\prime}=(\operatorname{lin} F)^{\perp}=\{y \in E:|x| \wedge|y|=0$, $\forall x \in \operatorname{lin} F\}$ (cf. [10, p. 38]). Since (lin $F)^{\perp}$ is a lattice ideal the set $G=K \cap(\operatorname{lin} F)^{\perp}$ is a face of $K$ disjoint from $F$, and hence $G \cong F^{\prime}$. However if $x \in F^{\prime} \backslash G$ then there exists a $y \in F$ such that $x \wedge y=z \neq 0$; but, since $F$ and $F^{\prime}$ are faces of $K$ and $x=z+(x-z), y=z+(y-z)$, this implies that $z /\|z\| \in F \cap F^{\prime}$ which is impossible. Therefore we have $(\operatorname{lin} F)^{\perp}=\operatorname{lin} G=\operatorname{lin} F^{\prime}$.

If $E$ is complete in its norm then it is an $A L$-space. If $F$ is norm-closed then the continuity of the lattice operations in $E$ shows that lin $F$ is also norm-closed, and hence is a band. Therefore, by a theorem of Riesz (cf. [10, p. 39]), lin $F$ has an order-direct complement in $E$, and so $K$ is uniquely decomposable at $F$. If, conversely, $K$ is uniquely decomposable at $F$ then there exists a natural affine function $f$ on $K$ such that $F=f^{-1}(0), F^{\prime}=f^{-1}(1)$. The function $f$ has an obvious extension to a continuous linear functional $g$ on $E$ and, since $F=K \cap g^{-1}(0)$, it follows that $F$ is norm-closed.

If $K$ is a compact simplex then $E$ is certainly a Banach space, and so the following result is immediate.

COROLlary. If $K$ is a compact simplex and $F$ a face of $K$, then $K$ is uniquely decomposable at $F$ if and only if $F$ is norm-closed.

The corollary generalizes Alfsen's result that a compact simplex is uniquely decomposable at each closed (i.e., compact) face. When $K$ is an arbitrary compact convex set Alfsen and Andersen [2] characterize the decomposable faces of $K$. However it is not true that every linearly compact simplex is decomposable at every norm-closed face, as the following example shows.

Example. Let $K$ denote the continuous nonnegative functions $f$ on 


$$
[0,1] \text { such that } \int_{0}^{1} f(t) d t=1 \text {, and let } F=\left\{f \in K: \int_{0}^{1 / 2} f(t) d t=0\right\} \text {. }
$$

Then $K$ is a base for the lattice cone in $C[0,1]$, and hence is a linearly compact simplex, and it is clear that $F$ is a face of $K$. The norm in $C[0,1]$ associated with $K$ is the $L_{1}[0,1]$-norm, and hence $F$ is norm-closed. Suppose that there exists a face $F^{\prime}$ complementary to $F$ in $K$. Then, since $\mathrm{f}(1 / 2)=0$ for all $f \in F$, there exists a $u \in F^{\prime}$ such that $u(1 / 2)>0$. However it is easy to decompose $u$ nontrivially $u=\lambda g+(1-\lambda) h$ with $g \in F, h \in F^{\prime}$ and $0<\lambda<1$. Since $F^{\prime}$ is a face of $K$ it follows that $g \in F \cap F^{\prime}$, which is a contradiction. Therefore $K$ is not decomposable at the norm-closed face $F$.

It has been shown by Asimow [4] that the state space of a function algebra is decomposable at every extreme point, and so such a property does not characterize simplexes among compact convex sets ; this property does however characterize simplexes among finitedimensional compact convex sets as the following slightly more general result shows.

Proposition. If $K$ is a compact convex set which is decomposable at each extreme point $x$, and such that each complementary face $\{x\}^{\prime}$ is closed, then $K$ is a finite-dimensional simplex.

Proof. If the set $K_{e}$ of extreme points of $K$ is infinite then there exists an accumulation point $u \in K$. For each $x \in K_{e}$ the set $K_{e}$ consists of $x$ together with the extreme points of the closed set $\{x\}^{\prime}$. Consequently $u \in\{x\}^{\prime}$ for all $x \in K_{e}$. Therefore the intersection of the faces $\{x\}^{\prime}$ forms a closed face $F$ of $K$ which is not empty, since $u \in F$. However if $\mathrm{y}$ is an extreme point of $F$ then $y \in K_{e}$, and also $y \in\{y\}^{\prime}$ which is impossible. Hence $K_{e}$ is finite.

If $K_{e}$ has $m$ points and $K$ has dimension $n$ then, for each $x \in K_{e}$, it is clear that $\{x\}^{\prime}$ has $m-1$ extreme points and has dimension $n-1$, and $\{x\}^{\prime}$ has a similar decomposition property to $K$. Reducing in this way we see eventually that $m=n+1$, that is $K$ is an $n$-dimensional simplex.

If $K$ is a compact simplex then the above result shows that not all faces $\{x\}^{\prime}$ can be closed. For example, for the simplex $\left\{x \in l_{1}\right.$ : $x \geqq 0,\|x\| \leqq 1\}$ all but one of the faces $\{x\}^{\prime}$ are closed, while for the simplex of probability measures on $[0,1]$ none of the faces $\{x\}^{\prime}$ are closed.

2. We prove an analogue for linearly compact simplexes of Edwards' separation theorem [6], which characterizes compact simplexes; this is a corollary of the following result. 
THeorem 2. Let $P$ be a cone possessing the Riesz decomposition property, and let $-f, g$ be subadditive homogeneous functionals on $P$ with $f \geqq g$. Then there exists an additive homogeneous functional $h$ on $P$ such that $f \geqq h \geqq g$.

Proof. If we define $h$ on $P$ by

$$
h(x)=\inf \left\{\sum_{i=1}^{n} f\left(x_{i}\right): x=\sum_{i=1}^{n} x_{i}, x_{i} \in P\right\}
$$

then it is clear that $f \geqq h \geqq g$, and hence $h$ is finite-valued. Moreover, $h$ is positive-homogeneous and subadditive. If $x=y+z$ with $x, y, z \in P$, and if $\varepsilon>0$ choose $x_{i} \in P$ such that $x=\sum_{i=1}^{n} x_{i}$ and $\sum_{i=1}^{n} f\left(x_{i}\right) \leqq h(x)+\varepsilon$. Then there exist $a_{i j} \in P$ such that $\sum_{i=1}^{n} a_{i 1}=$ $y, \sum_{i=1}^{n} a_{i 2}=z, a_{i 1}+a_{i 2}=x_{i}$ for $i=1,2, \cdots, n$. We have

$$
h(x) \geqq \sum_{i=1}^{n} f\left(x_{i}\right)-\varepsilon \geqq \sum_{i=1}^{n} f\left(a_{i 1}\right)+\sum_{i=1}^{n} f\left(a_{i 2}\right)-\varepsilon \geqq h(y)+h(z)-\varepsilon,
$$

so that $h$ is additive and homogeneous.

In the corollary below $K$ will denote a linearly compact subset of $E$, again contained in a hyperplane $e^{-1}(1)$ and such that lin $K=E$. By $A^{b}(K)$ we will denote the Banach space of all bounded real-valued affine functions on $K$ with the supremum norm. If $\operatorname{co}(K \cup-K)$ is. linearly bounded then its Minkowski functional is a norm in $E$ and $A^{b}(K)$ is simply the Banach dual space of $E$ for this norm. In the particular case when $K$ is compact for some locally convex Hausdorff topology on $E, A^{b}(K)$ is the second dual space of $A(K)$.

COROLlary. The following statements are equivalent.

(i) $K$ is a linearly compact simplex.

(ii) $\operatorname{co}(K \cup-K)$ is linearly compact and, if $-f, g$ are bounded. convex functions on $K$ with $f \geqq g$, there exists an $h \in A^{b}(K)$ such that $f \geqq h \geqq g$.

Proof. (i ) $\rightarrow$ (ii). That co $(K \cup-K)$ is linearly compact was. proved in [5, Th. 2]. If $P$ is the cone generated by $K$ as a base then $P$ is a lattice-cone. If $f$ and $g$ are extended homogeneously to the rest of $P$ then the existence of the required $h \in A^{b}(K)$ follows from the theorem.

(ii) $\rightarrow$ ( i ). If $u_{1}, u_{2}, v_{1}, v_{2} \in A^{b}(K)$ and $u_{1}, u_{2} \leqq v_{1}, v_{2}$ then, putting. $\mathrm{g}(x)=\max \left[u_{1}(x), u_{2}(x)\right], f(x)=\min \left[v_{1}(x), v_{2}(x)\right]$ for all $x \in K$, we obtain a function $h \in A^{b}(K)$ such that $u_{1}, u_{2} \leqq h \leqq v_{1}, v_{2}$. The $w^{*}$-compactness of order intervals in $A^{b}(K)$ now shows that $A^{b}(K)$ is a vector lattice, in fact an $A M$-space. Therefore $E$ is an $A L$-space and, in 
particular, each $\eta \in E$ has a unique decomposition $\eta=\eta_{1}-\eta_{2}$ with $\eta_{i} \geqq 0$ and $\|\eta\|=\left\|\eta_{1}\right\|+\left\|\eta_{2}\right\|$, namely for $\eta_{1}=\eta^{+}, \eta_{2}=\eta^{-}$. Since, by hypothesis, co $(K \cup-K)$ is the closed unit ball of $E$ it follows that $E$ is a sublattice of $E$. Therefore $K$ is a linearly compact simplex.

It is perhaps surprising that the linear compactness condition on co $(K \cup-K)$ cannot be dropped, as the following two simple examples show.

Examples. (i) Let $E$ be the linear subspace of $l_{1}$ spanned by those elements with only finitely many nonzero coordinates, together with the two elements $\left\{2^{-n}\right\},\left\{(-3)^{-n}\right\}$, and let $K=\{x \in E: x \geqq 0$, $\left.\|x\|_{1} \leqq 1\right\}$. If $S=\left\{x \in E:\|x\|_{1} \leqq 1\right\}$ then it is obvious that for each $\varepsilon>0$ we have co $(K \cup-K) \leqq S \subseteq(1+\varepsilon) \operatorname{co}(K \cup-K)$. If $x=\left\{\left(-3^{-n}\right\}\right.$ then $x^{+} \notin E$, so that $2 x \in S$ but $2 x \notin$ co $(K \cup-K)$. Therefore co $(K \cup-K)$ is not linearly closed; in other terminology $E$ has a $(1+\varepsilon)$ generating cone for all $\varepsilon>0$ but not a 1-generating cone. However, a straightforward verification shows that $E$ has the Riesz decomposition property and hence, by Theorem $2, K$ has the separation property stated in part (ii) of the Corollary. However $K$ is not a simplex.

(ii) Let $K$ denote the polynomials $p$ nonnegative on $[0,1]$ and such that $\int_{0}^{1} p(x) d x=1$. It is clear that co $(K \cup-K)$ is not linearly compact because the polynomials do not constitute a sublattice of $L_{1}[0,1]$. It is true, but less obvious, that lin $K$ has the Riesz decomposition property (of. [7]). We are grateful to Professor W. A. J. Luxemburg for bringing this fact and reference to our notice.

By an ordered Banach space we shall mean a partially ordered Banach space which has a closed, normal, generating cone. If $X$ is an ordered Banach space then so is $X^{*}$ (cf. [8]). The following lemma now follows from a result of Kadison ([9, Lemma 4.3]).

Lemma 1. Let $X$ be an ordered Banach space and let

$$
K=\left\{f \in X^{*}: f \geqq 0,\|f\| \leqq 1\right\},
$$

equipped with the $w^{*}$-topology. Then $X$ is order and topologically isomorphie to

$$
A_{0}(K) \equiv\{f \in A(K): f(0)=0\}
$$

LEMMA 2. Let $C$ be a cone in a vector space $V$, let $p$ be a function homogeneous on $C$ and let $f$ be a function affine on $V$ such that $f(x) \leqq p(x)$ for all $x \in C$. Then the linear function $g=f-f(0)$ satisfies $g(x) \leqq p(x)$ for all $x \in C$. 
Proof. It is simple to check that $g$ is linear on $V$. Suppose that there is a point $x \in C$ such that $g(x)>p(x)$. Then if $\varepsilon=-f(0)$ and $\delta=f(x)-p(x)$ we have $\varepsilon \geqq 0$ and $g(x)-p(x)=\delta+\varepsilon>0$. Hence there exists an $r \geqq 1$ such that $r(\delta+\varepsilon)>\varepsilon$, and we have

$$
f(x)=r^{-1} f(r x)+\left(1-r^{-1}\right) f(0) .
$$

Therefore

$$
f(r x)-p(r x)=r(f(x)-p(x))+(r-1) \varepsilon=r(\delta+\varepsilon)-\varepsilon>0,
$$

which gives a contradiction.

The following theorem is the main result of this section and is a topological version of Theorem 2 .

THEOREM 3. Let $X$ be an ordered Banach space such that the dual cone $P^{*}$ is a lattice cone in $X^{*}$, and let $-f, g$ be $w^{*}$-continuous subadditive homogeneous functionals on $P^{*}$ with $f \geqq g$. If either (i) $f=u_{1} \wedge u_{2}, g=v_{1} \vee v_{2}$, where $u_{1}, u_{2}, v_{1}, v_{2} \in X$, or (ii) the dual cone in $X^{* *}$ possesses an interior point, then there exists an $h \in X$ such that $f \geqq h \geqq g$.

Proof. If $K=\left\{x \in P^{*}:\|x\| \leqq 1\right\}$ then Lemma 1 shows that we can assume that $X=A_{0}(K)$, and it is sufficient to find an $h \in X$ such that $f(x) \geqq h(x) \geqq g(x)$ for all $x \in K$.

Let $G$ denote the $w^{*}$-closed convex hull of the graph of $f$ in $K \times R$ and define $\hat{f}(x)=\sup \{u(x): u \in A(K), u \leqq f\}$ for all $x \in K$. A straightforward calculation shows that $\hat{f}(x) \leqq \inf \{r:(x, r) \in G\}$ for each $x \in K$. If $\mu<\inf \{r:(x, r) \in G\}$ then by separating $(x, \mu)$ from $G$ we obtain a $v \in A(K)$ such that $v \leqq f$ while $v(x)>\mu$; therefore $\hat{f}(x)=\inf$ $\{r:(x, r) \in G\}$. Given $\varepsilon>0$, for each $x \in K$ let $N_{x}$ be a $w^{*}$-compact convex neighbourhood of $x$ such that $|f(x)-f(y)|<\varepsilon$ for each $y \in N_{x}$, and let $K \subseteq \bigcup_{i=1}^{n} N_{x_{i}}$. For each $x \in K$ we therefore have

$$
(x, \hat{f}(x)) \subseteq \operatorname{co~} \bigcup_{i=1}^{n}\left\{N_{x_{i}} \times\left[f\left(x_{i}\right)-\varepsilon, f\left(x_{i}\right)+\varepsilon\right]\right\},
$$

and so we can write $x=\sum_{i=1}^{n} \lambda_{i} y_{i}, \hat{f}(x)=\sum_{i=1}^{n} \lambda_{i} r_{i}$ with $y_{i} \in N_{x_{i}}$ and $r_{i} \in\left[f\left(x_{i}\right)-\varepsilon, f\left(x_{i}\right)+\varepsilon\right]$ for each $i$. If we now define for each $x \in P^{*}$

$$
\bar{f}(x)=\inf \left\{\sum_{i=1}^{n} f\left(x_{i}\right): x_{i} \in P^{*}, \sum_{i=1}^{n} x_{i}=x\right\}
$$

then, for each $x \in K$,

$$
\bar{f}(x) \leqq \sum_{i=1}^{n} \lambda_{i} f\left(y_{i}\right) \leqq \sum_{i=1}^{n} \lambda_{i} f\left(x_{i}\right)+\varepsilon \leqq \sum_{i=1}^{n} \lambda_{i} r_{i}+2 \varepsilon=\hat{f}(x)+2 \varepsilon .
$$


Therefore $\bar{f}(x) \leqq \hat{f}(x)$ for each $x \in K$. If $\alpha>0$ and if $\hat{f}_{\alpha}(x)=\sup$ $\{u(x): u \in A(\alpha K), u \leqq f\}$ then the argument shows that $\bar{f}(x) \leqq \hat{f}_{\alpha}(x)$ for all $x \in \alpha K$; in particular $\hat{f}_{\alpha}(0)=0=\bar{f}(0)$.

If condition (i) holds then we have

$$
\bar{f}(x)=\inf \left\{u_{1}\left(x_{1}\right)+u_{2}\left(x_{2}\right): x=x_{1}+x_{2}, x_{i} \in P^{*}\right\} .
$$

Since $P^{*}$ is a normal cone we can choose $\alpha>0$ such that $\left\|x_{1}\right\|+\left\|x_{2}\right\| \leqq \alpha\|x\|$ whenever $x=x_{1}+x_{2}$ with $x_{i} \in P^{*}$.

If condition (ii) holds and if $\zeta$ is an interior point of the dual cone in $X^{* *}$ then the order interval $[-\zeta, \zeta]$ is the unit ball for an equivalent norm in $X^{* *}$, and hence $X^{*}$ has an equivalent norm which is additive on $P^{*}$. Therefore there exists an $\alpha>0$ such that $\sum_{i=1}^{n}\left\|x_{i}\right\| \leqq \alpha\left\|\sum_{i=1}^{n} x_{i}\right\|$ whenever $x_{i} \in P^{*}$.

Now let $x=\sum_{i=1}^{n} x_{i} \neq 0$, where $x_{i} \in P^{*}$, and with $n=2$ if (i) holds. If $\lambda=\sum_{i=1}^{n}\left\|x_{i}\right\|$, and if $y_{i}=0$ when $x_{i}=0, y_{i}=\lambda x_{i}\left\|x_{i}\right\|$ when $x_{i} \neq 0$, then $y_{i} \in \alpha K$ for each $i$. Since $\hat{f}_{\alpha}$ is convex on $\alpha K$ we have

$$
\begin{aligned}
\hat{f}_{\alpha}(x) & =\hat{f}_{\alpha}\left(\sum_{i=1}^{n} \frac{\left\|x_{i}\right\|}{\lambda} y_{i}\right) \leqq \sum_{i=1}^{n} \frac{\left\|x_{i}\right\|}{\lambda} \hat{f}_{\alpha}\left(y_{i}\right) \leqq \sum_{i=1}^{n} \frac{\left\|x_{i}\right\|}{\lambda} f\left(y_{i}\right) \\
& =\sum_{i=1}^{n} f\left(x_{i}\right) .
\end{aligned}
$$

In case (ii) this inequality gives $\hat{f}_{\alpha}(x) \leqq \bar{f}(x)$ for each $x \in K$, while in case (i) we have $\hat{f}_{\alpha}(x) \leqq u_{1}\left(x_{1}\right)+u_{2}\left(x_{2}\right)$ which again gives $\hat{f}_{\alpha}(x) \leqq \bar{f}(x)$; in either case therefore we have proved that $\bar{f}(x)=\hat{f}_{\alpha}(x)$ for each $x \in K$. If we define $\|f\|=\sup \{|f(x)|: x \in K\}$, and $\|\bar{f}\|$ similarly, then we have $|\bar{f}(x)| \leqq \sum_{i=1}^{n}\|f\|\left\|x_{i}\right\| \leqq \alpha\|f\|\|x\|$ for each $x \in K$, so that $\|\bar{f}\| \leqq \alpha\|f\|$.

By Theorem $2 \bar{f}$ is additive on $P^{*}$ and the above argument shows that $\bar{f}$ is $w^{*}$-l. s. c. on $\beta K$ for each $\beta>0$. The set $\left\{x \in P^{*}: \bar{f}(x) \leqq r\right\}$ is convex and its intersection with each multiple of the unit ball of $X^{*}$ is $w^{*}$-closed; hence $\bar{f}$ is $w^{*}$-l. s. c. on $P^{*}$. If we write $\bar{g}$ for $-(-\bar{g})$ then $\bar{g}$ is $w^{*}$-u.s. c. on $P^{*}$ and is additive, homogeneous and satisfies $g \leqq \bar{g} \leqq \bar{f} \leqq f$. If $\varepsilon>0$ and $r>\alpha$ then, by separating the sets $\left\{(x, t) \in P^{*} \times R ; t>\bar{f}(x)\right\}$ and $\{(y, s-\varepsilon / r) \in K \times R: s \leqq \bar{g}(y)\}$ and applying Lemma 2, we obtain a $w_{\varepsilon} \in X$ such that $w_{\varepsilon} \leqq f$ and $w_{\varepsilon}(x)>g(x)-\varepsilon / r$ for all $x \in K$. Hence if $z_{\varepsilon}=\left(g-w_{\varepsilon}\right) \vee 0, z_{\varepsilon}$ is homogeneous, subadditive and $\mathrm{w}^{*}$-continuous on $P^{*}$ with $\left\|z_{e}\right\|<\varepsilon / r$. The above argument shows that $\bar{z}_{\varepsilon}$ is $w^{*}$-u. s. c. on $P^{*}$ and that $\left\|\bar{z}_{\varepsilon}\right\| \leqq \alpha\left\|z_{\varepsilon}\right\|<\varepsilon$. Since the set $K \times\{\varepsilon / r\}$ is disjoint from the $w^{*}$ closed cone $\left\{(x, t): x \in P^{*}, t \leqq \bar{z}_{\epsilon}(x)\right\}$ the separation theorem gives a $p_{\varepsilon} \in X$ such that $p_{\varepsilon} \geqq \bar{z}_{\varepsilon} \geqq g-w_{\varepsilon}, 0$ and $\left\|p_{\varepsilon}\right\| \leqq \varepsilon$.

Using the procedure of the preceding paragraph choose $f_{1}, g_{1} \in X$ 
such that $f_{1} \leqq f, g_{1} \geqq 0, g \leqq f_{1}+g_{1}$ and $\left\|g_{1}\right\|<1 / 2$, in particular we have $f \wedge\left(f_{1}+g_{1}\right) \geqq g \vee f_{1}$. By induction there exist sequences $\left\{f_{n}\right\}$ and $\left\{g_{n}\right\}$ in $X$ such that (a) $g_{n} \geqq 0$, $\left\|g_{n}\right\|<2^{-n}$, (b) $g \vee f_{n} \leqq f_{n+1}+g_{n+1}$, (c) $f_{n+1} \leqq f \wedge\left(f_{n}+g_{n}\right)$. Properties (b) and (c) give $-g_{n+1} \leqq f_{n+1}-f_{n} \leqq g_{n}$ so that $\left\|f_{n+1}-f_{n}\right\|<2^{-n}$. Therefore $\left\{f_{n}\right\}$ converges to $h \in X$ such that $h \leqq f$ by (c), and $h \geqq g$ by (b).

Corollary 1 (Andô [3]). If $X$ is an ordered Banach space such that $X^{*}$ is a lattice for the dual ordering then $X$ has the Riesz decomposition property.

COROLlary 2 (Edwards [6]). If $K$ is a compact simplex and if $-f, g$ are u. s. c. convex functions on $K$ with $f \geqq g$ then there exists an $h \in A(K)$ such that $f \geqq h \geqq g$.

Proof. By truncating if necessary we may assume that $f$ and $g$ are bounded, say $|f(x)|,|g(x)| \leqq \lambda$ for all $x \in K$. First suppose that the strict inequality $f>g$ holds ; then the set $G=\{(x, t): \lambda \leqq t \leqq g(x)\}$ is compact in $K \times R$ and is a subset of the convex set $H=\{(y, s): s<f(y)\}$ which is relatively open in $K \times R$. Therefore, taking the convex hull of a finite covering of $G$ by compact convex neighbourhoods in $H$, we see that $H$ contains the closed convex hull of $G$. Hence for each $x \in K$ there is an $f_{x} \in A(K)$ and a neighbourhood $U_{x}$ of $x$ such that $g<f_{x}$ while $f_{x}(y)<f(y)$ for all $y \in U_{x}$. If $K \leqq \bigcup_{i=1}^{n} U_{x_{i}}$ and if $f^{\prime}=$ $f_{x_{1}} \wedge \cdots \wedge f_{x_{n}}$ then $f^{\prime}$ is continuous and concave on $K$ with $g<f^{\prime}<f$. Similarly we can construct a continuous convex function $g^{\prime}$ on $K$ such that $g<g^{\prime}<f^{\prime}<f$. The functions $-f^{\prime}, g^{\prime}$ have natural extensions to $\mathrm{w}^{*}$-continuous subadditive homogeneous functions on the positive cone $P^{*}$ of $A(K)^{*}$ such that $g^{\prime} \leqq f^{\prime}$, and so Theorem 3 gives an $h^{\prime} \in A(K)$ such that $g<g^{\prime} \leqq h^{\prime} \leqq f^{\prime} \leqq f$.

In the general case $f \geqq g$ there exists an $h_{1} \in A(K)$ such that $f+1>h_{1}>g-1$. By considering the functions $\left(f \wedge h_{1}\right)+1 / 2$ and $\left(g \vee h_{1}\right)-1 / 2$ we similarly obtain an $h_{2} \in A(K)$ such that $f+1 / 2>h_{2}>g-1 / 2$ while $\left\|h_{2}-h_{1}\right\|<1 / 2$. Proceeding in this way we obtain a sequence $\left\{h_{n}\right\}$ which converges in $A(K)$ to $h$ such that $g \leqq h \leqq f$.

\section{REFERENCES}

1. E. M. Alfsen, On the decomposition of a Choquet simplex into a direct convex sum of complementary faces, Math. Scand. 17 (1965), 169-176.

2. E. M. Alfsen and T. B. Andersen, Split faces of compact convex sets, Aarhus Universitet, Preprint Series, 1968/69, No. 32.

3. T. Andô, On fundamental properties of a Banach space with a cone, Pacific J. Math. 12 (1962), 1163-1169. 
4. L. Asimow, Directed Banach spaces of affine functions, Trans. Amer. Math. Soc. 143 (1969), 117-132.

5. D. A. Edwards, On the homeomorphic affine embedding of a locally compact cone into a Banach dual space endowed with the vague topology, Proc. London Math. Soc. (3) 14 (1964), 399-414.

6. Séparation des fonctions réelles définies sur un simplexe de Choquet, C. R. Acad. Sci. Paris 261 (1965), 2798-2800.

7. L. Fuchs, Riesz vector spaces and Riesz algebras, Queens Papers in Pure and Applied Math. No. 1, 1966.

8. J. Grosberg and M. Krein, Sur la décomposition des fonctionelles en composantes positives, C. R. (Doklady) de 1'Acad. Sci. de 1'URSS 25 (1939), 723-726.

9. R. V. Kadison, Transformations of states in operator theory and dynamics, Topology, suppl. 2 (1965), 177-198.

10. A. L. Peressini, Ordered topological vector spaces, Harper and Row, New York, 1967.

Received July 8, 1969. The first author was partially supported by NSF Grant GP-11475.

California, los Angeles

California Institute of TeChNology 



\title{
PACIFIC JOURNAL OF MATHEMATICS
}

\author{
EDITORS
}

\author{
H. SAMELSON \\ Stanford University \\ Stanford, California 94305 \\ RichaRd PIERCE \\ University of Washington \\ Seattle, Washington 98105
}

J. DugundJI

Department of Mathematics

University of Southern California

Los Angeles, California 9.0007

RICHARD ARENS

University of California

Los Angeles, California 9.0024

\section{ASSOCIATE EDITORS}

E. F. BeCKenBACH
B. H. NeumanN

F. WoLE

K. YoSHIDA

\section{SUPPORTING INSTITUTIONS}

\author{
UNIVERSITY OF BRITISH COLUMBIA \\ CALIFORNIA INSTITUTE OF TECHNOLOGY \\ UNIVERSITY OF CALIFORNIA \\ MONTANA STATE UNIVERSITY \\ UNIVERSITY OF NEVADA \\ NEW MEXICO STATE UNIVERSITY \\ OREGON STATE UNIVERSITY \\ UNIVERSITY OF OREGON \\ OSAKA UNIVERSITY \\ UNIVERSITY OF SOUTHERN CALIFORNIA
}

\author{
STANFORD UNIVERSITY \\ UNIVERSITY OF TOKYO \\ UNIVERSITY OF UTAH \\ WASHINGTON STATE UNIVERSITY \\ UNIVERSITY OF WASHINGTON \\ AMERICAN MATHEMATICAL SOCIETY \\ CHEVRON RESEARCH CORPORATION \\ TRW SYSTEMS \\ NAVAL WEAPONS CENTER
}

The Supporting Institutions listed above contribute to the cost of publication of this Journal, but they are not owners or publishers and have no responsibility for its content or policies.

Mathematical papers intended for publication in the Pacific Journal of Mathematics should be in typed form or offset-reproduced, (not dittoed), double spaced with large margins. Underline Greek letters in red, German in green, and script in blue. The first paragraph or two must be capable of being used separately as a synopsis of the entire paper. The editorial "we" must not be used in the synopsis, and items of the bibliography should not be cited there unless absolutely necessary, in which case they must be identified by author and Journal, rather than by item number. Manuscripts, in duplicate if possible, may be sent to any one of the four editors. Please classify according to the scheme of Math. Rev. Index to Vol. 39. All other communications to the editors should be addressed to the managing editor, Richard Arens, University of California, Los Angeles, California, 90024.

50 reprints are provided free for each article; additional copies may be obtained at cost in multiples of 50 .

The Pacific Journal of Mathematics is published monthly. Effective with Volume 16 the price per volume (3 numbers) is $\$ 8.00$; single issues, $\$ 3.00$. Special price for current issues to individual faculty members of supporting institutions and to individual members of the American Mathematical Society: $\$ 4.00$ per volume; single issues $\$ 1.50$. Back numbers are available.

Subscriptions, orders for back numbers, and changes of address should be sent to Pacific Journal of Mathematics, 103 Highland Boulevard, Berkeley, California, 94708.

PUBLISHED BY PACIFIC JOURNAL OF MATHEMATICS, A NON-PROFIT CORPORATION

Printed at Kokusai Bunken Insatsusha (International Academic Printing Co., Ltd.), 7-17, Fujimi 2-chome, Chiyoda-ku, Tokyo, Japan. 


\section{Pacific Journal of Mathematics}

\section{Vol. 34, No. $2 \quad$ June, 1970}

Shair Ahmad, On the oscillation of solutions of a class of linear fourth order

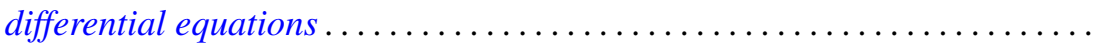

Leonard Asimow and Alan John Ellis, Facial decomposition of linearly

compact simplexes and separation of functions on cones ..............

Kirby Alan Baker and Albert Robert Stralka, Compact, distributive lattices of

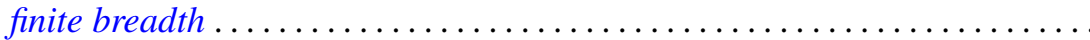

James W. Cannon, Sets which can be missed by side approximations to

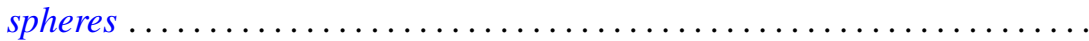

Prem Chandra, Absolute summability by Riesz means .................. 335

Francis T. Christoph, Free topological semigroups and embedding topological semigroups in topological groups....

Henry Bruce Cohen and Francis E. Sullivan, Projecting onto cycles in smooth, reflexive Banach spaces.................................

John Dauns, Power series semigroup rings .......................

Robert E. Dressler, A density which counts multiplicity ................

Kent Ralph Fuller, Primary rings and double centralizers ................

Gary Allen Gislason, On the existence question for a family of products.......

Alan Stuart Gleit, On the structure topology of simplex spaces .............

William R. Gordon and Marvin David Marcus, An analysis of equality in

certain matrix inequalities. $I \ldots \ldots \ldots \ldots \ldots \ldots \ldots \ldots$

Gerald William Johnson and David Lee Skoug, Operator-valued Feynman

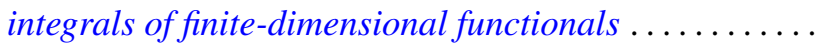

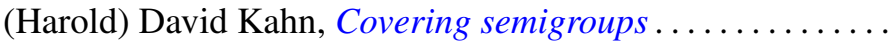

Keith Milo Kendig, Fibrations of analytic varieties

Norman Yeomans Luther, Weak denseness of nonatomic measures on perfect,

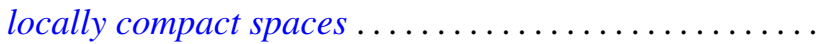

Guillermo Owen, The four-person constant-sum games; Discriminatory solutions on the main diagonal ...

Stephen Parrott, Unitary dilations for commuting contractions

Roy Martin Rakestraw, Extremal elements of the convex cone $A_{n}$ of

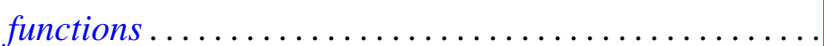

Peter Lewis Renz, Intersection representations of graphs by

William Henry Ruckle, Representation and series summability of complete

biorthogonal sequences.

F. Dennis Sentilles, The strict topology on bounded sets ...

Saharon Shelah, A note on Hanf numbers ...

Harold Simmons, The solution of a decision problem for several classes of rings. . .

Kenneth S. Williams, Finite transformation formulae involving the Legendre 